\title{
Stereoscopy and refocusing from axial scanning in 3D imaging
}

\section{Estereoscopía y reenfoque a partir del escaneo axial en imaginería 3D}

\author{
J. R. Alonso* and J. A. Ferrari \\ Instituto de Física, Facultad de Ingeniería, Universidad de la República, \\ J. Herrera y Reissig 565, Montevideo, Uruguay \\ ${ }^{(*)}$ E-mail: julialon@fing.edu.uy \\ Received: 01/12/2016 Accepted: 08/08/2017 \\ DOI: $10.7149 /$ OPA.50.3.49040
}

\begin{abstract}
:
In the present work we use a monocular camera along with an electrically tunable lens (ETL) to acquire a multi-focus stack without mechanical displacements, avoiding vibrations and misregistration during imaging acquisition. It is possible to decode and reconstruct new images of interest (images that are not part of the original acquired stack) by digital processing algorithms with a physical basis. We show how Fourier domain operations along with the knowledge of the focal distances employed for sweeping the optical axis, enable the redistribution of the information encoded in the original stack. In this way, from the multi-focus stack, it is possible to refocus digitally the scene, with synthetically reshaped apertures (changing its shape and size), obtaining extended-depth-of-field as a particular case (i.e., as captured with a pin-hole camera). We can also consider displacements of the pinhole for shifting the viewer's perspective of the 3D scene and obtaining stereoscopic pairs of images (left and right eyes images) as particular cases. Finally, it is also possible to combine both implementations and reconstruct the scene as captured from a different perspective and with a different aperture at the same time.
\end{abstract}

Key words:Three-dimensional image processing; Image reconstruction techniques; Computational optical imaging.

\section{RESUMEN:}

En el presente trabajo, usamos una cámara monocular junto a una lente de foco ajustable eléctricamente (ETL) para adquirir un stack multifoco sin desplazamientos mecánicos, evitando vibraciones y problemas de registro durante en la adquisición. Es posible decodificar y reconstruir nuevas imágenes de interés a través de algoritmos de procesamiento digital con bases físicas. Operaciones en el dominio de Fourier en combinación con el conocimiento de las distancias focales empleadas para barrer el eje óptico, permiten la redistribución de la información en el stack original. De esta forma, a partir de un stack multifoco, es posible reenfocar digitalmente la escena, a través de aperturas sintetizadas digitalmente con diferentes formas y tamaños, obteniendo por ejemplo una imagen con la profundidad de campo extendida (cámara pinhole). También consideramos desplazamientos digitales del pinhole para cambiar la perspectiva de la escena 3D y obtener pares de imágenes estereoscópicas (imágenes para el ojo izquierdo y derecho) como caso particular. Finalmente, también es posible combinar ambas implementaciones y reconstruir la escena como si hubiera sido capturada tanto desde una perspectiva diferente como con una apertura diferente simultáneamente.

Palabras clave: Procesamiento de imágenes tridimensionales; Técnicas de reconstrucción de imagen; Imaginería óptica computacional. 


\section{REFERENCES AND LINKS / REFERENCIAS Y ENLACES}

[1] A. Kubota, K. Kodama, and K. Aizawa, "Registration and blur estimation methods for multiple differently focused images," In Proceedings of the International Conference on Image Processing ICIP, IEEE 2, 447-451 (1999). https://doi.org/10.1109/ICIP.1999.822936

[2] J. R. Alonso, A. Fernández, G. A. Ayubi and J. A. Ferrari, "All-in-focus image reconstruction under severe defocus," Opt. Lett. 40(8), 1671-1674 (2015). https://doi.org/10.1364/OL.40.001671

[3] J. W. Goodman, Introduction to Fourier optics. Roberts and Company Publishers (1996).

[4] A. Ben-Israel and T. N. Greville, Generalized inverses: theory and applications, volume 15. Springer Science \& Business Media. (2003).

[5] J. R. Alonso, A. Fernández and J. A. Ferrari, "Reconstruction of perspective shifts and refocusing of a three-dimensional scene from a multi-focus image stack," Appl. Opt. 55(9), 2380:2386 (2016).

https://doi.org/10.1364/A0.55.002380

\section{Introduction}

The depth of field (DOF) is the range of scene depths that appears acceptably sharp (in-focus) in an image for a given focal length and aperture of the optical system. DOF can be increased by making the aperture smaller (Fig. 1 (top)), however, this reduces the amount of light received by the detector, resulting in greater image noise. To avoid this problem, for scientific and biomedical applications as well as for photography or industrial machine vision, wide apertures are used (Fig. 1 (bottom)) but this limits the DOF of the optical system, and an axial scanning of a 3D scene is usually performed. Once a multi-focus z-stack is acquired through the axial scanning, a post-acquisition computational image processing is nedded to, for example, obtain a synthesized image with extended depth of field (EDOF).

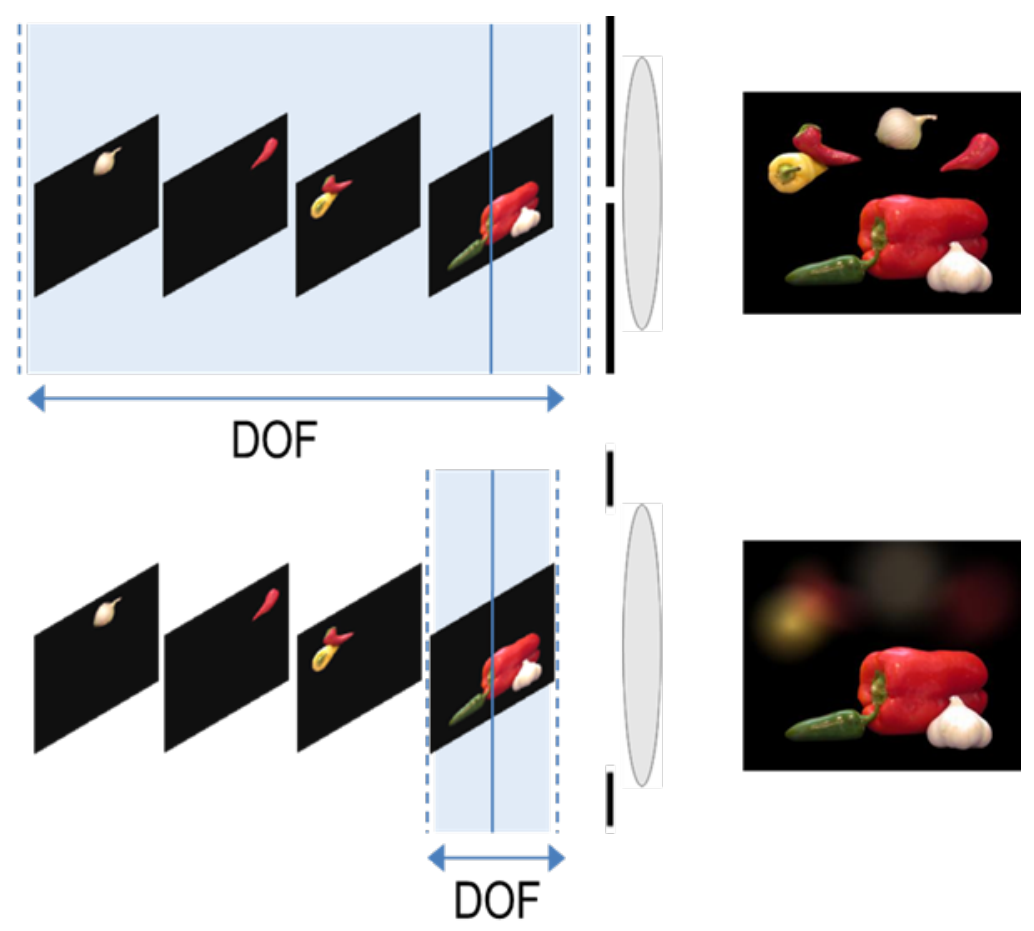

Fig.1. (Top) narrow aperture and large DOF; (bottom) wide aperture and shallow DOF .

\section{Multi-focus image acquisition and image formation model}

Conventional strategies to perform axial scanning and acquiring multiple differently focused images of a 3D scene lead to differences in scale or alignment between the images. Sometimes these differences can be ignored but for thick objects (e.g. thick specimens in microscopy), a pre-processing procedure, known as registration, need to be done to correct the differences [1]. In [2], a simple optical system which 
incorporates an electrically focus-tunable lens (ETL) and does not require registration was considered. This calibrated optical system behaves as a thin lens with an effective focal length that can be varied by changing the electrical current through the ETL. In this section we describe the image formation model as a piecewiseplanar 3D aproximation, i.e. the set of acquired images can be modeled as a set of $2 \mathrm{D}$ convolutions of the piecewise-planar object with depth-variant PSFs.

Each image of the z-stack contains both in-focus and out-of-focus contributions. The $i_{k}$ image $(k=1, \ldots, N)$ taken with the optical system focusing at axial distance $z=z_{k}$ can be described by:

$$
i_{k}(x, y)=f_{k}(x, y)+\sum_{k^{\prime} \neq k} h_{k k^{\prime}}(x, y) * f_{k^{\prime}}(x, y),
$$

Where $f_{k}$ is the in-focus region of $i_{k}$ and the part of the scene that looks defocused comes from the 2D convolution between $f_{k^{\prime}}$ and the 2D intensity Point-Spread Function (PSF) $h_{k k^{\prime}}(x, y)$ associated with the depths $z_{k}$ and $z_{k^{\prime}}$ :

$$
h_{k k^{\prime}}(x, y)=\frac{1}{\pi r_{k k^{\prime}}^{2}} \operatorname{circ}\left(\frac{\sqrt{x^{2}+y^{2}}}{r_{k k^{\prime}}}\right), \quad \frac{r_{k k^{\prime}}}{p}=R_{0}\left|\frac{1}{z_{k}}-\frac{1}{z_{k^{\prime}}}\right| .
$$

where $R_{0}=R d / p$ and $p$ is the pixel pitch of the camera and $r_{k k}$, is the radius of the blur circle. In the present work, $\mathrm{R}$ is the radius of the imaging lens, and $d$, is the distance from this lens to the imaging plane. In Eq. (2), the values of those parameters are: $2 \mathrm{R}=13.5 \mathrm{~mm}, \mathrm{~d}=16 \mathrm{~mm}$ and $\mathrm{p}=5.65 \mu \mathrm{m}$.

As stated by the convolution theorem, the Fourier transform of a convolution is the product of Fourier transforms [3], then it is useful to consider the Fourier transform of Eq. (1):

$$
I_{k}(u, v)=F_{k}(u, v)+\sum_{k^{\prime} \neq k} H_{k k^{\prime}}(u, v) F_{k^{\prime}}(u, v),
$$

where $(u, v)$ are spatial frequencies, $H_{k k^{\prime}}$ are Optical Transfer Functions (OTFs) (i.e., $H_{k k^{\prime}}=F T\left\{h_{k k^{\prime}}\right\}$ ), while $I_{k}=F T\left\{i_{k}\right\}$ and $F_{k^{\prime}}=F T\left\{f_{k^{\prime}}\right\}$. The set of $N$ coupled equations in (3) can be arranged in vector form as:

$$
\left(\begin{array}{c}
I_{1}(u, v) \\
I_{N}(u, v)
\end{array}\right)=\left(\begin{array}{ccc}
1 & H_{12}(u, v) & H_{1 N}(u, v) \\
& 1 & \\
& & H_{N-1 N}(u, v) \\
& & 1
\end{array}\right)\left(\begin{array}{l}
F_{1}(u, v) \\
F_{N}(u, v)
\end{array}\right),
$$

with $N$-element column vectors $I, F$ and $N \mathrm{x} N$ symmetric matrix $H\left(H_{k^{\prime} k}=H_{k k^{\prime}}\right)$ :

$$
I(u, v)=H(u, v) F(u, v) .
$$

\section{Novel viewpoints and stereoscopic pairs of images synthesis}

As $H(u, v)$ may not be invertible, e.g. as it is the case for the DC frequency component $(u, v)=(0,0)$, then a solution to the system may be found through the Moore-Penrose pseudo-inverse [4]. The Moore-Penrose pseudo-inverse provides the set of vectors that minimize the Euclidean norm $\|H(u, v) F(u, v)-I(u, v)\|$ in the least squares sense, among which the minimun norm vector is given by

$$
F(u, v)=H^{\dagger}(u, v) I(u, v) .
$$

When $H(u, v)$ is invertible then $H^{\dagger}(u, v)=H^{-1}(u, v)$, and Eq. (5) reduces to $F(u, v)=H^{-1}(u, v) I(u, v)$. We are interested in those linear combinations of the elements of the minimum norm vectors in Eq. (5) that lead to the reconstruction of novel viewpoints. This can be synthesized by simulating the displacement of a pinhole camera in the $(x, y)$ plane. Each in-focus region $f_{k}(x, y)$ should be shifted in an amount according to the disparity associated with the focusing distance $z_{k}$ and the virtual baseline displacement vector $b_{x}$ (in $\mathrm{mm}$ ) of a pinhole camera [5]: 


$$
s_{b_{x}}(x, y)=\sum_{k=1}^{N} f_{k}\left(x-\frac{d}{z_{k}} b_{x}, y\right) .
$$

By means of Fourier transform shift theorem [3], which states that a translation in space domain introduces a linear phase shift in the frequency domain, we obtain the Fourier transform of Eq. (6):

$$
S_{b_{x}}(u, v)=\sum_{k=1}^{N} \mathrm{e}^{-j \frac{2 \pi d}{z_{k}}\left(b_{x} u\right)}\left(H^{\dagger}(u, v) I(u, v)\right)_{k} .
$$

Note that for $b_{x}=0$ the inverse Fourier transform of Eq. (7) gives an image as captured through a centered pinhole, i.e. an image with extended depth-of-field (see Fig. 2(e)).
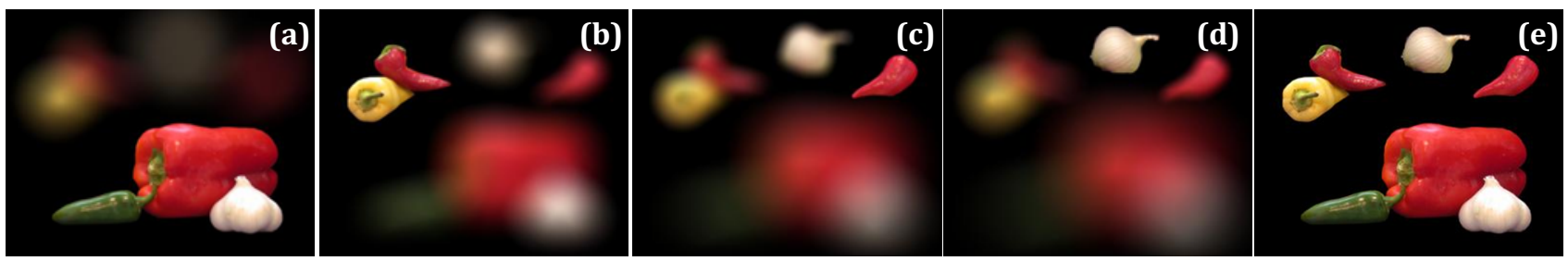

Fig.2. (a-d) Synthetic axial scanning for different focusing distances and (e) the all-in-focus image (i.e. with extended depth-of-field) reconstructed by the proposed method.

To synthesize a stereoscopic pair of images, we just need to shift the virtual pinhole camera to the left and to the right in the same amount i.e. for the left view $b_{x}=+\mathrm{B} / 2$ and for the right view $b_{x}=-\mathrm{B} / 2$ :

$$
\begin{gathered}
S_{B / 2}(u, v)=\sum_{k=1}^{N} e^{-j \frac{2 \pi d}{z_{k}}\left(\frac{B}{2} u\right)}\left(H^{\dagger}(u, v) I(u, v)\right)_{k} \\
S_{-B / 2}(u, v)=\sum_{k=1}^{N} e^{+j \frac{2 \pi d}{z_{k}}\left(\frac{B}{2} u\right)}\left(H^{\dagger}(u, v) I(u, v)\right)_{k}
\end{gathered}
$$

And finally, by inverse Fourier transforming, it is possible to obtain the left and right eye views:

$$
\begin{aligned}
& i_{L}(x, y)=F T^{-1}\left\{S_{B / 2}(u, v)\right\} \\
& i_{R}(x, y)=F T^{-1}\left\{S_{-B / 2}(u, v)\right\}
\end{aligned}
$$

It is worth to mention that the displacements of the viewpoint cannot exceed the actual size of the original circular aperture $\left(b_{x} \leq \mathrm{R}\right)$. Then the máximum interpupilary distance (B) is limited by $2 \mathrm{R}$.

In Fig. 3, a set of 2 images acquired by the optical system proposed in [2] is shown.
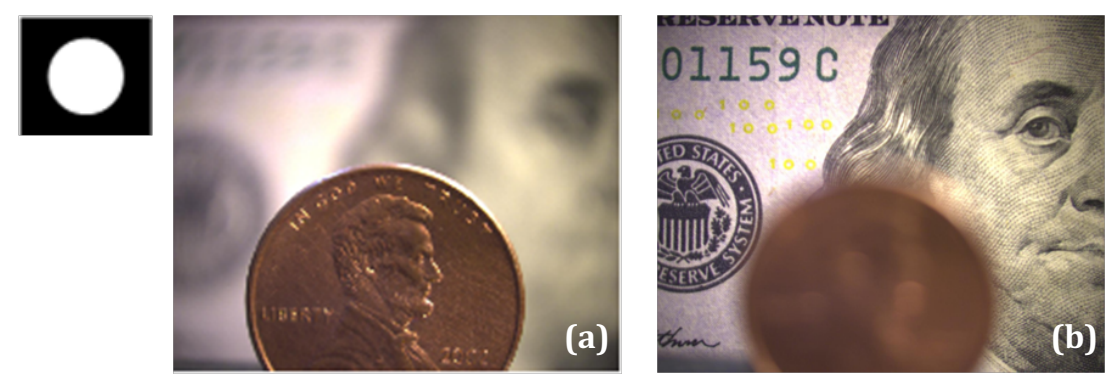

Fig.3. Set of real images acquired with an ETL+CCD camera optical system with a circular aperture. (a) System focusing on the foreground and (b) focusing on the background.

Figure 4(a-b) shows the right and left eye views for $b_{x}=-1.2 \mathrm{~mm}$ and $b_{x}=+1.2 \mathrm{~mm}$ respectively. In Fig. 4(c) the red/cyan anaglyph is shown (red/cyan glasses are needed to visualize it). 

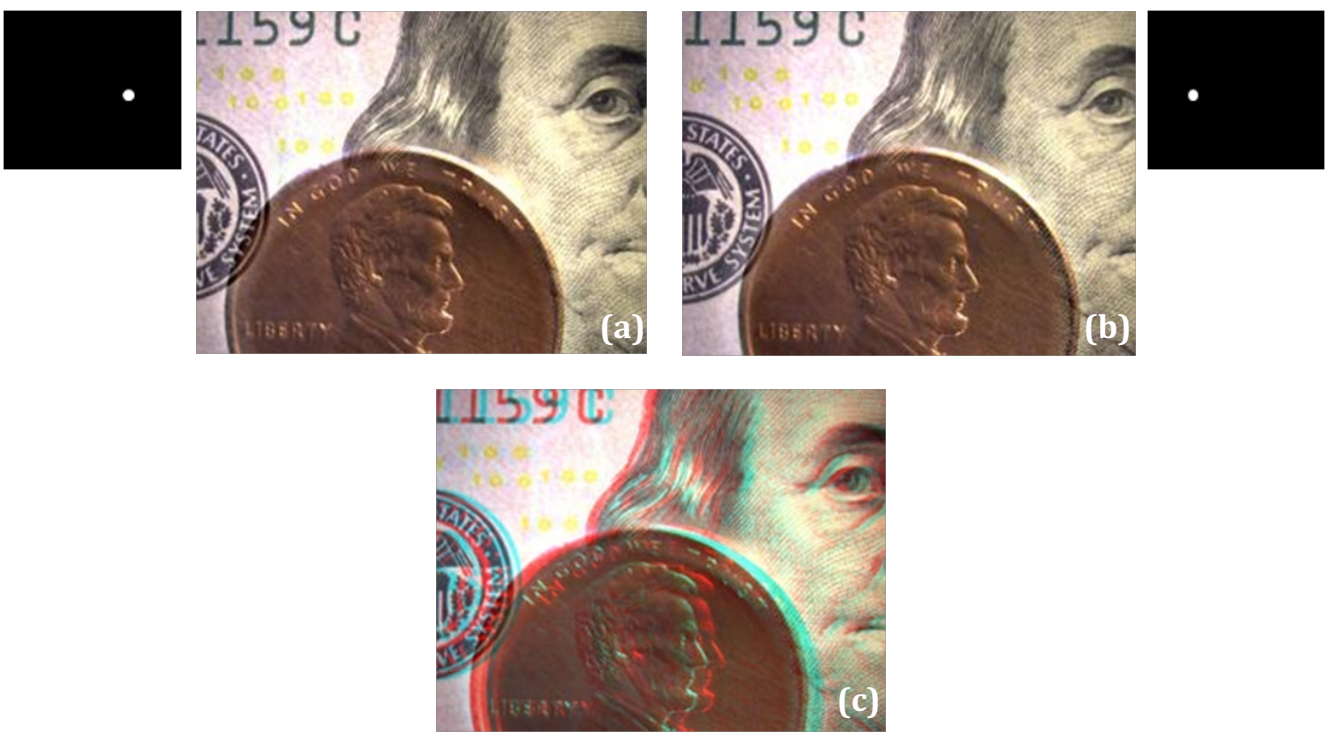

Fig.4. (a) Pinhole shifted to the right ( $b x=-1.2 \mathrm{~mm})$, (b) pinhole shifted to the left $(b x=+1.2 \mathrm{~mm})$. The right and left views (a,b) are arragend for cross-eyed view in order to perceive depth. Alternatively in (c) the red/cyan anaglypgh is presented (red/cyan glasses nedded).

\section{Novel viewpoints and aperture reshaping}

It is also possible to synthesize images as if they were captured through different apertures (shape and size) than that of the original circular one. The extended depth-of-field image can be seen as a centered pinhole aperture as we already mentioned but other aperture shapes are also possible.

Images acquired through a different aperture will have a different set of PSFs related to its shape and size given by, let's say $\left\{g_{k k^{\prime}}(x, y)\right\}$. Note that for the particular case of a pinhole aperture, $g_{k k^{\prime}}(x, y)=\delta(x, y)$.

Then, an image captured whith the system focusing at $z_{k}$ with a different displaced aperture, will be given, instead of Eq. (1), by

$$
i_{k}^{(g)}(x, y)=f_{k}(x, y)+\sum_{k^{\prime} \neq k} g_{k k^{\prime}}(x, y)^{*} f_{k^{\prime}}(x, y)
$$

where,

$$
g_{k k^{\prime}}(x, y)=g_{k k^{\prime}}(x, y) * \delta\left(x-\frac{d b_{x}}{z_{k}}, y-\frac{d b_{y}}{z_{k}}\right) .
$$

Then, Fourier transforming Eq. (11) and considering the Moore-Penrose pseudo-inverse:

$$
I_{k}^{(g)}(u, v)=F_{k}(u, v)+\sum_{k^{\prime} \neq k} G_{k k^{\prime}}(u, v)\left(H^{\dagger}(u, v) I(u, v)\right)_{k^{\prime}},
$$

and finally by inverse Fourier transforming the novel viewpoint from a different aperture is obtained:

$$
i_{k}^{(g)}(x, y)=F T^{-1}\left\{I_{k}^{(g)}(u, v)\right\}
$$

In Fig. 5, the result from Eq. (14) for a synthesized annular shifted aperture is shown. The PSF, $\mathrm{g}_{\mathrm{kk}}(\mathrm{x}, \mathrm{y})$, corresponding to the annular aperture can be obtained considering the substraction of two circular apertures of different radius. For Fig. 5 the external and internal radius of the annular aperture considered are $\mathrm{R} / 2$ and $2 \mathrm{R} / 5$ respectively. 

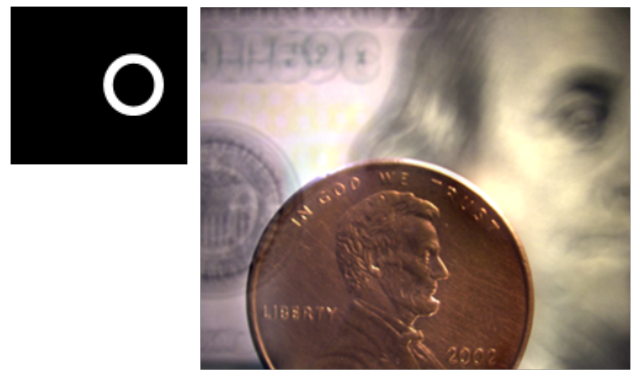

Fig.5. Synthetized image with a different point of view $(\mathrm{bx}=-1.5 \mathrm{~mm})$ and a different aperture (annular aperture with half of the radius $\mathrm{R}$ of the original circular aperture).

\section{Conclusions}

In the present work we have showed how Fourier domain operations enable the synthesis of new images of interest from an acquired multi-focus image stack like an image with extended-depth-of-field or an image of the scene as if captured under a different aperture (in shape or size). We also showed how to reconstruct images as seen under different perspectives and stereo pairs of images as a particular case. Finally, the reconstruction of the scene as captured from a different perspective and with a different aperture at the same time was also synthesized.

\section{Acknowledgements}

The authors acknowledge financial support from Programa de Desarrollo de las Ciencias Básicas (PEDECIBA, Uruguay), Agencia Nacional de Investigación e Innovación (ANII, Uruguay) and Comisión Sectorial de Investigación Científica (CSIC, UdelaR, Uruguay). 